\title{
Deutschland in der europäischen Krise: Teil der Lösung oder Teil des Problems?
}

Deutschlands Rolle in der gegenwärtigen Krise der Eurozone wird höchst kontrovers beurteilt. Während die einen die deutsche Wirtschaft als einen Anker der Sicherheit betrachten und sich Sorgen darüber machen, welche Belastungen insbesondere den deutschen Steuerzahlern beim Retten von „Schuldensündern“ noch zuzumuten seien, sehen andere in Deutschland den Hauptsünder und werfen den Industriegewerkschaften vor, durch das Zulassen eines Unterbietungswettbewerbs bei den Lohnkosten andere Länder unter Druck zu setzen. Um zu einem Urteil in dieser Streitfrage zu kommen, betrachtet der Beitrag die großen Veränderungen im deutschen Wirtschafts- und Sozialmodell während der vergangenen zehn bis 20 Jahre. ${ }^{1}$

\section{1 \\ Vorbemerkung}

In den turbulenten Tagen vor dem EuroKrisengipfel Ende Oktober erklärte der SPD-Fraktionsvorsitzende im Deutschen Bundestag: „Die Zerrüttung dieser Regierung wird zu einer Hypothek für Europa, aber Europa darf an dieser Regierung nicht scheitern“ (FTD, 27. 10. 2011). „Gut gebrüllt, Löwe!“, möchte man Frank-Walter Steinmeier da zurufen, bliebe nicht das ungute Gefühl, dass das krisenverschärfende Taktieren der Bundesregierung nur ein Teil der Hypothek ist, die der Europäischen Union (EU) von Deutschland aufgebürdet wird. Vielleicht sogar der kleinere Teil: Das noch viel größere Problem ist das grundlegende „Geschäftsmodell“ Deutschlands innerhalb der EU und vor allem der Eurozone - ein Modell, an dessen Durchsetzung nicht zuletzt einige führende heutige Oppositionspolitiker tatkräftig mitgewirkt haben. Diese These möchte ich im Folgenden erläutern, indem ich zunächst den Umbruch im deutschen Modell des Kapitalismus vor der Krise (Abschnitt 2) und während der dramatischen Rezession (Abschnitt 3) skizziere. Ich konzentriere mich dabei auf die grundlegenden Veränderungen des Beschäftigungsmodells, also der Institutionen, die das Lohnarbeitsverhältnis prägen und die Rückwirkungen dieser Veränderungen auf Europa. Im Anschluss daran (Abschnitt 4) werfe ich einen Blick auf die Bedeutung dieses Umbruchs für die gegenwärtige Krise der Eurozone. Der Beitrag schließt mit einem Ausblick (Abschnitt 5).

\subsection{DEUTSCHLANDS GESCHÄFTS- MODELL IM EURORAUM}

\section{Vor der Krise: „Hartz IV vergiftet Europa ${ }^{\mu}$}

Der Kern dessen, was über lange Zeit als die deutsche Variante des „koordinierten“ oder "Rheinischen Kapitalismus“ bezeichnet wurde, war eine Kombination von wirtschaftlicher Dynamik und relativ geringer sozialer Ungleichheit. Bis vor rund 20 Jahren halfen Institutionen wie das System der industriellen Beziehungen, das Arbeitsrecht und der Wohlfahrtsstaat dabei, die auf hoher Wertschöpfung und Qualitätsproduktion beruhenden Erfolge der weltmarktorientierten, von "geduldigem Kapital“ und gut ausgebildeten Beschäftigten getragenen Industrie der gesamten Volkswirtschaft und auch großen Teilen der Gesellschaft zugute kommen zu lassen. Auf die ruckartig vollzogene monetäre Angliederung Ostdeutschlands folgten jedoch anderthalb Jahrzehnte eines tief greifenden politischen und wirtschaftlichen Umbruchs, der in seiner Radikalität über die Anpassungen an den neoliberalen Mainstream in den meisten anderen EU-Ländern noch hinausging (vgl. Lehndorff et al. 2009). Im Ergebnis bot das deutsche Modell am Vorabend der großen Krise 2008/09 ein neues Bild: eine wieder erstarkte und hoch leistungsfähige Exportindustrie in einem Umfeld zunehmender sozialer und institutioneller Desintegration und Fragmentierung. Diese widersprüchliche Konstellation wird im Folgenden näher beleuchtet, um zum besseren Verständnis zweier Fragen beizutragen: Wie wurde von Deutschland aus der Boden für die Krise mit bereitet, und welchen Anteil hat Deutschland am Ausbruch und Andauern der Euro-Krise?
Ausgangspunkt der Analyse ist die schwache Lohnentwicklung in Deutschland: Nirgendwo sonst in der EU war der Anstieg der durchschnittlichen Arbeitnehmerentgelte seit Beginn des Jahrzehnts bis zur Krise so gering wie in Deutschland. Die Lohnstückkosten stagnierten, im verarbeitenden Gewerbe gingen sie sogar etwas zurück (Niechoj et al. 2011). Soweit, so unstrittig. Nicht einig sind sich auch kritische Beobachter jedoch in zwei Fragen, die sich aus dieser Bestandsaufnahme ergeben: Haben insbesondere die Industriegewerkschaften durch eine zurückhaltende Lohnpolitik im Rahmen internationaler Standortkonkurrenz zu dieser Entwicklung beigetragen? Und: Welche Bedeutung hatte die Stagnation der Durchschnittslöhne in Deutschland und die damit einhergehende Steigerung seiner preislichen Wettbewerbsfähigkeit für das massive Anwachsen der Ungleichgewichte im Euroraum im zurückliegenden Jahrzehnt - und damit für das Entstehen der gegenwärtigen Krise?

\footnotetext{
1 Dieser Aufsatz beruht auf einem Beitrag für das demnächst beim Europäischen Gewerkschaftsinstitut in englischer Sprache erscheinende Buch über die Auswirkungen der Krise in zehn europäischen Ländern (Lehndorff 2012). Eine Veröffentlichung des Bandes in deutscher Sprache ist in Vorbereitung.
}

Steffen Lehndorff, Dr., ist Mitarbeiter der Forschungsabteilung Arbeitszeit und Arbeits organisation am Institut Arbeit und Qualifikation (IAQ) der Universität Duisburg-Essen. Arbeitsschwerpunkte: Arbeitszeiten, industrielle Beziehungen und nationale Beschäftigungssysteme im europäischen Vergleich. e-mail: steffen.lehndorff@uni-due.de 
Zunächst zur zweiten Frage: Selbstverständlich haben die Exporterfolge der deutschen Industrie (mehr als ein Drittel der deutschen Warenexporte entfallen auf den Fahrzeug-, den Maschinen- und den Anlagenbau) starke Fundamente in der hohen Spezialisierung und Produktqualität, der Serviceorientierung der Unternehmen und der Flexibilität und Qualifikation der Beschäftigten. Ebenso selbstverständlich jedoch haben, wie es die Deutsche Bundesbank (2011a, S. 17) zurückhaltend formuliert, die deutschen Exporterfolge im zurückliegenden Jahrzehnt von der „Verbesserung der preislichen Wettbewerbsfähigkeit Impulse“ erhalten (vgl. dazu auch European Commission 2010). ${ }^{2}$ Die auf produktbezogenen Fundamenten beruhende Wettbewerbsfähigkeit wurde also durch die Steigerung der preislichen Wettbewerbsfähigkeit ergänzt und verstärkt. Zugleich war mit der Währungs- union, innerhalb derer Deutschland rund zwei Fünftel seines Außenhandels betreibt, der Wechselkurs-Anpassungsmechanismus abgeschafft worden, der zuvor für periodischen Ausgleich gesorgt hatte. So war es kein Zufall, dass der beeindruckende Anstieg der Leistungsbilanzüberschüsse mit der Einführung des Euro begann (Abbildung 1).

Die andere Seite derselben Medaille: Das außenwirtschaftliche Ungleichgewicht beruhte auf einem binnenwirtschaftlichen Defizit Deutschlands. Im Zeitraum zwischen 2001 und 2008 waren drei Viertel des deutschen Wirtschaftswachstums auf den Exportüberschuss zurückzuführen, während die Binnennachfrage lediglich einen Beitrag von einem Viertel lieferte (Priewe/Rietzler 2010, S. 64). Ursache dafür war die schwache Lohnentwicklung als Bestandteil einer rasch zunehmenden Ungleichheit in der

\section{Abb. 1: Leistungsbilanzsalden Deutschlands mit den Ländern des Euro-Raums - in Mrd. $€-$}

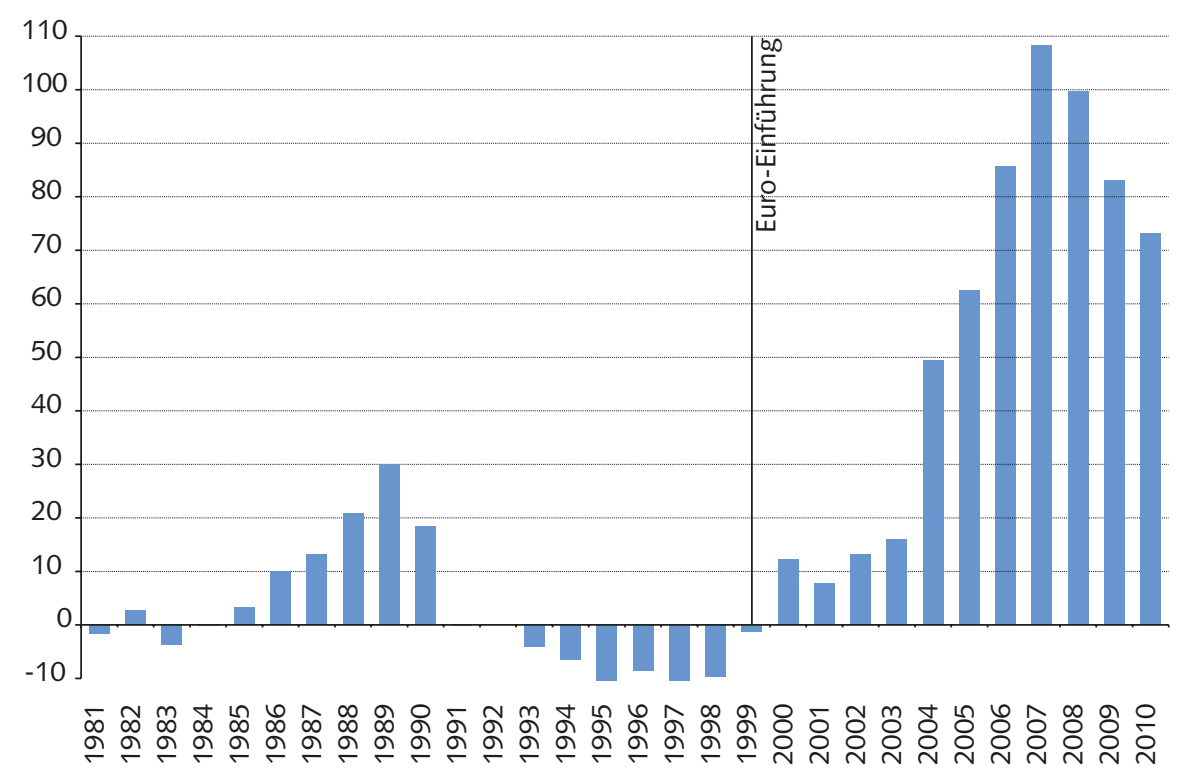

Quelle: Deutsche Bundesbank (o.J.); Joebges et al. (2010); Berechnungen des Autors.

WSI MITTEILUNGeN
Einkommens- und Vermögensverteilung (ich komme darauf zurück). Dieser Aspekt blieb übrigens auch der Deutschen Bundesbank (2011a, S. 33) nicht verborgen, die darauf aufmerksam machte, dass ein Teil der durch die Steigerung der preislichen Wettbewerbsfähigkeit entstandenen Kostenvorteile „offensichtlich auch zur Erhöhung der Gewinnmargen genutzt" wurde. Die Stagnation der Durchschnittslöhne verhinderte also sowohl eine Übertragung der vom Exportboom ausgehenden Wachstumsimpulse auf den Binnenmarkt als auch eine damit einhergehende Stärkung der Importe, mit der die exportinduzierten Wachstumsimpulse für die deutsche Industrie an das Ausland zurückgegeben worden wären (Joebges et al. 2010, S. 10). Diese Sonderrolle Deutschlands innerhalb der EU spiegelt sich wider in den Wachstumsraten des Bruttoinlandsprodukts (BIP), des Preisniveaus und der verfügbaren Haushaltseinkommen seit 2001: Nahezu durchgängig lagen die deutschen Steigerungsraten unter dem EU-Durchschnitt (Tabelle 1).

Wenn es richtig ist, dass die in diesen Daten zum Ausdruck kommenden Ungleichgewichte ihre wichtigste Ursache in der zunehmenden Ungleichheit in der Einkommens- und Vermögensverteilung in Deutschland haben, dann führt die kontroverse Diskussion darüber, ob Deutschland zu viel exportiere, nicht wirklich weiter. Sinnvoller fände ich es, die andere Seite derselben Medaille ins Zentrum zu stellen: Deutschland importiert $z u$ wenig. Innerhalb einer Währungsunion muss der schwache Binnenmarkt ihrer größten Volkswirtschaft zu einem Problem für die

2 Die Europäische Zentralbank (ECB 2011) beziffert die Verbesserung der preislichen Wettbewerbsfähigkeit Deutschlands gegenüber den wichtigsten Welthandelsländern im Zeitraum 1999 bis Anfang 2011 auf 16 \% (Basis: GDP deflator). Die einzigen übrigen Länder der Europäischen Währungsunion mit positiven Werten sind Finnland $(6,4 \%)$, Österreich (4,6\%) und Frankreich (3,2\%).

Tabelle 1: Reales Wachstum, Inflationsrate und verfügbare Haushaltseinkommen: Deutschland und EU-27 (2001 - 2008) - jährliche Steigerungsraten in \% -

\begin{tabular}{|c|c|c|c|c|c|c|c|c|c|c|c|c|c|c|c|c|}
\hline & \multicolumn{2}{|c|}{2001} & \multicolumn{2}{|c|}{2002} & \multicolumn{2}{|c|}{2003} & \multicolumn{2}{|c|}{2004} & \multicolumn{2}{|c|}{2005} & \multicolumn{2}{|c|}{2006} & \multicolumn{2}{|c|}{2007} & \multicolumn{2}{|c|}{2008} \\
\hline & $\mathrm{DE}$ & EU & $\mathrm{DE}$ & EU & $\mathrm{DE}$ & EU & $\mathrm{DE}$ & EU & $\mathrm{DE}$ & EU & $\mathrm{DE}$ & EU & $\mathrm{DE}$ & EU & $\mathrm{DE}$ & EU \\
\hline Reales BIP & 1,2 & 2,0 & 0,0 & 1,2 & $-0,2$ & 1,3 & 1,2 & 2,5 & 0,8 & 2,0 & 3,2 & 3,2 & 2,5 & 2,9 & 1,3 & 0,8 \\
\hline Inflation & 2,0 & 3,2 & 1,4 & 2,5 & 1,0 & 2,1 & 1,7 & 2,3 & 1,6 & 2,3 & 1,6 & 2,3 & 2,3 & 2,4 & 2,6 & 3,7 \\
\hline $\begin{array}{l}\text { Verfügbares } \\
\text { Einkommen }\end{array}$ & 2,1 & 3,3 & $-0,4$ & 1,6 & 0,5 & 1,6 & 0,2 & 1,4 & 0,6 & 1,7 & 1,0 & 1,7 & $-0,2$ & 1,6 & 0,6 & 1,5 \\
\hline
\end{tabular}


Entwicklung aller Mitgliedsländer werden. Das deutsche Wachstums- und Geschäftsmodell ist nicht nachhaltig.

Doch was verbirgt sich - und damit komme ich zurück zur ersten Frage - hinter der schwachen Lohnentwicklung in Deutschland, die häufig etwas irreführend auch als „Lohnmäßigung“ bezeichnet wird? Der Versuch, dies zu verstehen, führt ins Zentrum der Umbrüche im deutschen Beschäftigungsmodell in den zurückliegenden zwei Jahrzehnten.

\section{2 "LOHNMÄßIGUNG" - WAS STECKT DAHINTER?}

Wer die Entwicklung der Tariflöhne von 2000 bis 2010 betrachtet, wird eine vielleicht überraschende Feststellung machen. Ausgerechnet in der Metall- und der Chemieindustrie, also den beiden Wirtschaftszweigen, die von allen Branchen am intensivsten der internationalen Konkurrenz ausgesetzt sind, stiegen die tarifvertraglich vereinbarten Löhne und Gehälter am stärksten. Zwar ist es den vergleichsweise starken und durchsetzungsfähigen Gewerkschaften in diesen Branchen nicht gelungen, die fortschreitende Einkommensumverteilung zulasten der abhängig Beschäftigten zu verhindern, doch immerhin schöpften die Tariflohnerhöhungen ungefähr den sogenannten ,verteilungsneutralen“ Spielraum aus, der als Summe von Preissteigerungen und gesamtwirtschaftlichem Produktivitätswachstum definiert wird (Bispinck 2011). Dass es in exportorientierten Branchen immer wieder Elemente eines Wettbewerbs-Korporatismus gibt, ist schwer zu bestreiten, doch die noch wichtigeren Ursachen der geringen durchschnittlichen Tariflohnsteigerungen sind offenbar in solchen Branchen zu suchen, die in geringerem Maße oder gar nicht der internationalen Konkurrenz ausgesetzt sind, wie dem Einzelhandel und vor allem dem öffentlichen Dienst, der das Schlusslicht der großen Branchen bildet.

Dies macht auf den ersten Strukturbruch aufmerksam, nämlich die Demontage der traditionellen Architektur der deutschen Version von „pattern bargaining", also des Geleitzugprinzips in Tarifverhandlungen, bei denen zumeist die IG Metall die Marken setzte. In dem Maße, wie dieses Prinzip an Wirkung verloren hat, ist die Kluft zwischen den Arbeitskosten in Industrie und Dienstleistungen (mit weiter zunehmender Tendenz) so groß geworden wie in keinem anderen EULand. Dadurch hat sich eine breite Palette inländischer Vorleistungen für die Industrie, einschließlich solcher durch Steuern finanzierten des öffentlichen Dienstes, im Vergleich zum Ausland erheblich verbilligt (Horn et al. 2007).

Der zweite Strukturbruch äußert sich darin, dass die effektiven Lohnsteigerungen von 2000 bis 2010 im Durchschnitt fast $50 \%$ unter den tarifvertraglichen Lohnerhöhungen lagen (nominal 12,7 \% gegenüber 23,6 \%), sodass die Reallöhne pro Kopf um $4 \%$ zurückgingen (Bispinck 2011). Ursächlich dafür waren

- die Schwächung der Gewerkschaften: Ihr Netto-Organisationsgrad fiel von ungefähr $30 \%$ Mitte der 1990er Jahre auf rund $20 \%$ am Vorabend der Krise (ICTWSS Database 2011);

- der Rückgang der Tarifbindung: 1996 arbeiteten $70 \%$ der Beschäftigten in Westdeutschland in Betrieben mit Bindung an Branchentarifverträge, 2008 waren es noch $55 \%$ (in Ostdeutschland fiel der Anteil von $56 \%$ auf $40 \%$ ); berücksichtigt man außerdem die Firmentarifverträge, arbeiteten am Vorabend der Krise rund $60 \%$ der Beschäftigten in tarifgebundenen Unternehmen (Ellguth/Kohaut 2010);

- die Demontage des Instruments der Allgemeinverbindlicherklärung von Flächentarifverträgen (Bispinck/Schulten 2009); - die zahlreichen lokalen Abweichungen von Flächentarifverträgen seit dem Beginn der 2000er Jahre (Haipeter 2009).

Über diese institutionellen Veränderungen hinaus haben - drittens - auch die Fundamente der Institutionen tiefe Risse bekommen. Folgende Veränderungen stellen zwar keine deutsche Besonderheit dar, aber sie verstärken in Verbindung mit den soeben genannten institutionellen Umbrüchen die bremsende Wirkung auf die Arbeitseinkommen:

(1) Durch Outsourcing von Vorleistungen werden immer größere Teile der Industrie in Industrie- oder Dienstleistungsbetriebe mit niedrigeren Arbeitsstandards verschoben.

(2) Eine damit vergleichbare Wirkung geht von den Privatisierungen vormals öffentlich erbrachter Dienstleistungen aus. In Verbindung mit der EU-Dienstleistungsrichtlinie wurden - z.B. bei der Post - gro- ße Bereiche mit massiver NiedriglohnKonkurrenz geschaffen.

(3) Durch die Steuerreformen seit 1998 entstanden den öffentlichen Haushalten erhebliche Einnahmenverluste (2010 betrugen sie 2,5\% des BIP; Truger/Teichmann 2010). Davon ging ein massiver Druck auf die Löhne (und auch die Arbeitszeiten) der im öffentlichen Dienst Beschäftigten aus.

Ein machtvolles viertes Bleigewicht für die Entwicklung der Löhne sind die seit 2003 vorgenommenen Änderungen an der Architektur der Arbeitsmarktregulierung:

(1) Der mit Hartz IV verbundene Systemwechsel - massive Reduzierung der Arbeitslosenversicherung zugunsten eines Fürsorgesystems mit Bedürftigkeitsprüfung (Knuth 2010) - zwingt zur Annahme von Beschäftigungsangeboten mit niedrigen Arbeitsstandards und löste eine breite Verunsicherung aus, die in weiten Teilen des Arbeitsmarkts eine Sogwirkung nach unten bewirkt (Erlinghagen 2010).

(2) Mit der de-facto Aushebelung des europäischen Equal-pay-Rechtsgrundsatzes in der Leiharbeit wurde durch die Bundesregierung der Weg für Dumping-Tarifverträge in dieser Branche geebnet (Weinkopf/ Vanselow 2008). Seit 2000 verdoppelte sich die Zahl der Leiharbeitskräfte bis Dezember 2010 auf knapp $3 \%$ aller sozialversicherungspflichtig Beschäftigten (BA 2011). Leiharbeitskräfte verdienten 2006 im Durchschnitt pro Stunde nur etwas mehr als halb so viel wie Standard-Beschäftigte (Destatis 2009).

(3) Ein kräftiger Schub für die Ausweitung atypischer Beschäftigung geht von der öffentlichen Subventionierung von Niedriglöhnen und insbesondere der Förderung von geringfügiger Beschäftigung (Minijobs) aus. Rund 15 \% aller abhängig Beschäftigten (mehrheitlich Frauen) sind

3 Ein Kommentator der Financial Times Deutschland brachte den Zusammenhang auf folgenden Nenner: „In den zwei Jahren nach Hartz-IV-Einführung brachen die Lohnkosten je produzierter Einheit in Deutschland um unheimliche fast vier Prozent ein, nachdem sie bis 2005 gestiegen waren. Das ist umso verrückter, als zeitgleich die Arbeitslosigkeit um fast eine Million sank - was nach den Regeln von Angebot und Nachfrage höheren statt geringeren Lohndruck erzeugen müsste. Kaum erklärbar, außer durch den HartzSchock" (Fricke 2010). 
heute ausschließlich geringfügig beschäftigt, und zwei Drittel von ihnen beziehen einen Niedriglohn, obwohl eine deutliche Mehrheit von ihnen einen beruflichen Abschluss hat (Weinkopf 2009).

Die Bedeutung der Minijob-Regelung hängt eng mit dem Fortbestehen der konservativen Elemente des Wohlfahrtsstaats zusammen, die eine begrenzte Teilnahme von Frauen am Erwerbsleben teils erzwingen und teils fördern: Der Mangel an Kinderbetreuungsangeboten erschwert es vielen Frauen mit kleinen Kindern, ihre berufliche Tätigkeit rasch wieder aufzunehmen, während das Steuer- und Sozialversicherungssystem die ungleiche Verteilung von Erwerbseinkommen bei verheirateten Paaren finanziell attraktiv macht (Sachverständigenkommission 2011). Auch wenn dieses den 1950er Jahren verhaftete System immer stärker in die Kritik gerät und partielle Veränderungen (wie die Einführung eines Elterngeldes nach schwedischem Vorbild) eingeleitet wurden, steht seine im Grundsatz unangetastete Stabilität in bemerkenswertem Kontrast zu dem neoliberal inspirierten Reformeifer auf anderen Gebieten (vgl. dazu Bogedan in diesem Heft).

Das Zusammenwirken des Umbruchs im Institutionensystem des deutschen Arbeitsmarkts einerseits und der hartnäckigen Verteidigung des konservativen Geschlechtermodells im Wohlfahrtsstaat andererseits findet seinen markantesten Ausdruck im Wachstum des Niedriglohnsektors. Seit Mitte der 1990er Jahre ist die Zahl der Beschäftigten, die weniger als zwei Drittel des Medianlohns verdienen, um die Hälfte gestiegen; ihr Anteil betrug am Vorabend der Krise $22 \%$ aller Beschäftigten (Bosch/Kalina 2008). Es ist dieses Ausfransen der Löhne nach unten, das sich hinter dem Rückgang der (preisbereinigten) durchschnittlichen Löhne aller Arbeitnehmerinnen und Arbeitnehmer in den 2000er Jahren verbirgt. Die Reallohnsenkung war einmalig in Europa und auch für Deutschland ein Novum - zumal in einer Phase wirtschaftlichen Wachstums. „Hartz IV vergiftet Europa“ - so brachte ein Kommentator der Financial Times Deutschland den Zusammenhang auf den Punkt (Münchau 2010).

\subsection{KRISENTREIBER UNGLEICHHEIT}

Den stagnierenden oder sogar sinkenden Durchschnittslöhnen stand eine konti- nuierlich (wenn auch nicht überdurchschnittlich) steigende Arbeitsproduktivität gegenüber. Im Ergebnis sank die bereinigte Lohnquote seit Beginn des Jahrzehnts bis zum Vorabend der Krise um mehr als sechs Prozentpunkte. Die Dynamik zunehmender Ungleichheit gehörte zu den stärksten in der EU (ILO 2010).

Hinzu kam, dass die Veränderungen in der Primärverteilung weitaus weniger, als dies zuvor üblich war, durch die Sekundärverteilung abgeschwächt wurden. Ursache dafür waren die bereits erwähnten Steuerreformen der rot-grünen Bundesregierung, mit denen der Spitzensatz der Einkommensteuer ebenso wie die Steuern auf Kapitaleinkünfte und Unternehmensgewinne erheblich gesenkt wurden. Im Ergebnis nahm die Ungleichheit in der Wachstumsphase 2004 bis 2008 besonders stark zu: Leicht rückläufigen Nettolöhnen stand ein Anstieg der Gewinne und Kapitaleinkommen um fast $20 \%$ gegenüber (Brenke 2011).

Anders als von den neoliberal inspirierten Hauptakteuren in Ökonomie und Politik prophezeit, ging von der zunehmenden Ungleichheit kein nennenswerter Impuls für Investitionen und Wachstum aus (Priewe/Rietzler 2010). Angesichts des stagnierenden Binnenmarkts lag dies zwar nahe, doch weitaus mehr als der Privatsektor war der Staat für die Investitionsschwäche verantwortlich: Der Anteil der öffentlichen Investitionen am BIP blieb immer weiter hinter dem Durchschnitt in der EU zurück (Dullien/Schieritz 2011). Die Regierungen sparten den selbst geschaffenen Einnahmerückgängen hinterher - es war ein „Jahrzehnt der Entstaatlichung“ (Bofinger 2008).

Die massive Umverteilung zugunsten von Gewinnen und Kapitaleinkommen in Deutschland war damit das Kondensat jenes grundlegenden Umbruchs im deutschen Modell des Kapitalismus seit Mitte der 1990er Jahre, der im Kern als Wechselwirkung von drei einander verstärkenden Prozessen verstanden werden kann:

- erstens, die massive Schwächung der auf sozialen Ausgleich hinwirkenden Institutionen auf dem Arbeitsmarkt;

- zweitens, die Stärkung und relative Verselbstständigung des Finanzkapitals, die auch auf die Unternehmensführung zurückgewirkt und das einstmals „geduldige“ Kapital auch in Deutschland ungeduldiger gemacht hat;
- und drittens, die systematische Schwächung der regulierenden, ausgleichenden und investierenden, also vorsorgenden Rolle des Staates.

Ein derart fundamentaler Umbau der Architektur der größten Volkswirtschaft Europas musste erhebliche Außenwirkungen haben. Insbesondere unter den Bedingungen der Währungsunion trug er maßgeblich zum Entstehen der Krise bei: einerseits durch das beschriebene Zusammenspiel von stagnierendem Binnenmarkt und immer größeren außenwirtschaftlichen Ungleichgewichten, und andererseits dadurch, dass für das nicht profitabel investierbare Geldkapital Einsatzfelder außerhalb der inländischen Produktion von Gütern und konsumierbaren Dienstleistungen gesucht werden mussten (Horn et al. 2009). Zahlreiche Deregulierungen des Finanzsektors durch die SPD-geführte Bundesregierung (Huffschmid 2010) öffneten den deutschen Gewinnen und Vermögenseinkommen die Türen für eine aktive Teilnahme an den weltweit boomenden Finanzmärkten und insbesondere an der Finanzierung des von vor allem privater, teils staatlicher Verschuldung getriebenem starken Wachstums in europäischen Defizitländern. Es ist eine nur kleine journalistische Übertreibung, wenn ein Kommentator im britischen Guardian schreibt: „Germany blew the bubbles that popped up in the rest of Europe“ (Chakrabortty 2011).

Der deutsche Kapitalismus war nicht Opfer der Krise, sondern trug maßgeblich dazu bei, ihr den Boden zu bereiten. Wie aber gelang es ihm, die Krise so schnell zu überwinden und - zumindest dem Augenschein nach - sogar gestärkt aus ihr hervorzugehen?

\section{In der Krise: Das Wunder, das keins war}

Der Absturz der deutschen Wirtschaft ab September 2008 war wegen ihrer extremen Exportabhängigkeit besonders dramatisch und wurde nur von Ländern mit platzenden Finanz- und Immobilienblasen übertroffen. Umso verblüffender war die Stabilität auf dem deutschen Arbeitsmarkt. Was in den Jahren 2008 und 2009 passierte, lässt sich auf einen einfachen Nenner bringen: Während vor der Krise der Umbruch im deutschen Beschäftigungsmodell aktiv zum Entstehen 
der europäischen und weltwirtschaftlichen Ungleichgewichte beigetragen hatte, war die Stabilisierung des Arbeitsmarkts und der Wirtschaft während der Krise ausgerechnet einer Reaktivierung derjenigen Elemente des deutschen Modells zu verdanken, die den neoliberal inspirierten Demontageeifer der Jahre davor überlebt hatten.

Die Wiederbelebung alter Tugenden begann mit dem plötzlichen, wenn auch nur vorübergehenden, Beiseitelegen vieler bislang gepflegter neoliberaler Dogmen in der Regierungspolitik. Quasi über Nacht wurden entgegen allen zuvor gebetsmühlenartig wiederholten Glaubenssätzen umfangreiche Konjunkturprogramme aufgelegt. Die Bundestagswahlen von 2009 standen vor der Tür und jegliches Zögern beim Retten der „Realwirtschaft" wäre angesichts der Milliardenausgaben und -bürgschaften für die Rettung von Banken zum politischen Desaster für die beiden großen Regierungsparteien geworden.

Die Konjunkturprogramme der Regierung stützten sich auf die aktive Mitarbeit sowohl der Arbeitgeberverbände als auch der Gewerkschaften. Es entstand ein informeller „Krisen-Korporatismus“ (Urban 2012), dessen wichtigste unmittelbare Wirkung in der Abwendung eines massenhaften Beschäftigungsabbaus in der krisengeschüttelten Industrie bestand. Diese Notgemeinschaft schloss zwar - wie der starke Rückgang der Leiharbeit zeigte zum Schutz von Stammbelegschaften den Rückgriff auf externe Flexibilität keineswegs aus. Doch ein noch größeres Gewicht hatte die interne Flexibilität. Dies war neu, oder besser: wieder neu. Nur wenige Jahre zuvor, während der zurückliegenden Rezessions- und Stagnationsphase, hatten viele Unternehmen ihre Belegschaften derart ausgedünnt, dass sie Schwierigkeiten und Personalengpässe hatten, als die Konjunktur wieder ansprang. Diese Erfahrung war noch frisch, und um qualifiziertes Personal zu halten, wurden jetzt kurzfristig selbst erhebliche Produktivitätseinbußen in Kauf genommen (Herzog-Stein et al. 2011).

Die Renaissance der internen Flexibilität fand ihren markantesten Ausdruck in der erheblichen Verkürzung der pro Kopf geleisteten Arbeitszeit, die weithin als wichtigste Erklärung für die Kluft zwischen BIP-Einbruch und Beschäftigungsstabilität gilt (OECD 2010). Sie beruhte auf der Mobilisierung unterschiedlicher Ressourcen des deutschen Beschäftigungsmodells - zum Teil neu entstandener, vor allem aber traditioneller und in der Krise wiederentdeckter Stärken, die den Abbruch und Umbruch der Vorjahre überlebt hatten (vgl. dazu ausführlich: Bosch 2011; Lehndorff 2011). Die prominenteste Rolle spielten dabei die Kurzarbeit, die Tarifverträge zur Beschäftigungssicherung, der Abbau von Überstunden und die in den Vorjahren zum Teil sehr stark angewachsenen Guthaben auf Arbeitszeitkonten (Fuchs et al. 2010). Zu den weniger beachteten Ressourcen des deutschen Beschäftigungsmodells, die die Priorisierung interner Flexibilität während der Krise attraktiv für Arbeitgeber machten, gehörte jedoch auch - trotz aller seiner Probleme - das Berufsbildungssystem (Voss-Dahm 2011). Es trug maßgeblich dazu bei, dass die Arbeitslosenquote unter Jugendlichen zunächst nicht stark anstieg und bis 2011 sogar unter das Niveau von 2008 fiel. (Allerdings liegt der Anteil prekär beschäftigter Jugendlicher ähnlich wie in anderen europäischen Ländern deutlich über der durchschnittlichen Rate aller Altersgruppen; Destatis 2011).

Die Rückbesinnung auf traditionelle Stärken des deutschen Beschäftigungsmodells war sicher der wichtigste Grund für das sogenannte Beschäftigungswunder während der Krise. Dennoch machte sich der Umbruch der Vorjahre in der Krise bemerkbar. Der schnelle und starke Rückgang der Leiharbeit signalisierte, wie weit die Dualisierung des Arbeitsmarktes bereits fortgeschritten war. Es war nur der Kürze der Krise zu verdanken, dass sich dies noch nicht in der Gesamtbilanz der Arbeitsmarktprozesse zeigte. Nicht so offensichtlich, aber ebenso wichtig, ist die Dualisierung in Gestalt der viel gelobten Flexibilität der Arbeitszeiten. In der Krise wurde es vielfach als Wohltat empfunden, Arbeitszeit-Puffer abbauen zu können, doch diese Puffer waren ja zuvor zulasten eines stärkeren Beschäftigungswachstums aufgebaut worden - vor allem in Gestalt hoher Guthaben auf Arbeitszeitkonten (IAQ 2011). Die Widersprüche, Ungleichgewichte und Fragilitäten, die mit dem neoliberal inspirierten Umbruch Einzug ins deutsche Beschäftigungsmodell gehalten haben, wirkten in der Krise fort - und tun dies auch weiterhin.

Dieses Fortwirken zeigte sich zunächst (in paradoxer Weise) in der konjunkturellen Entwicklung. So plötzlich und radikal der Absturz in die Krise war, so rasch und zunächst kräftig setzte bereits in der zweiten Jahreshälfte 2009 der Aufschwung wie- der ein - das eine wie das andere vom Export getrieben. Noch hatten viele deutsche Industrieunternehmen gewissermaßen mit laufendem Motor den Gang der Ereignisse abgewartet. Vielfach war die Krise auch $\mathrm{zu}$ Rationalisierungen genutzt worden. Als die Auslandsnachfrage im 3. Quartal 2009 wieder anzog, waren die qualifizierten Stammbelegschaften noch an Bord, die Kurzarbeit wurde beendet, die entlassenen Leiharbeitskräfte wurden wieder zurückgeholt und die leer geräumten Arbeitszeitkonten konnten wieder aufgefüllt werden. Es konnte wieder losgehen - und wie viele zunächst glaubten: weitergehen wie vor der Krise. Doch dies erwies sich bald als Illusion. Das wirtschaftliche Umfeld hatte sich in kürzester Zeit gründlich verändert, und dies am stärksten in Europa.

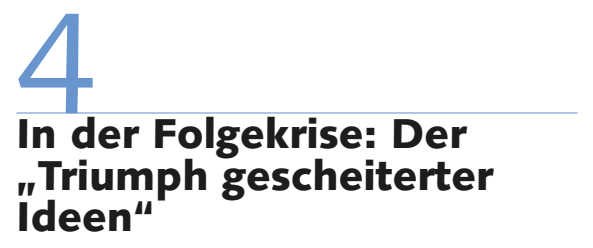

Die Wachstumsraten der deutschen Wirtschaft gehörten seit Ende 2009 zunächst zu den stärksten Europas, und Deutschland gilt auch weiterhin als Anker der Stabilität in einem Umfeld von Eurokrise und überbordenden Staatsschulden. Doch der Schein trügt erneut: Für eine Abkehr von dem hier analysierten Pfad der Untugend, der in die Krise führte, gibt es bislang keine Anzeichen. Auch für Deutschland gilt im Kern, was Paul Krugman (2010) mit Blick auf die USA als „den seltsamen Triumph gescheiterter Ideen“ bezeichnet hat: „Die Fundamentalisten des freien Marktes haben sich in allem geirrt - doch sie dominieren die politische Szene gründlicher als jemals zuvor“ (ebd.). Und doch muss ein wesentlicher Unterschied zur Zeit vor 2008 (und vielleicht auch zu den USA) festgehalten werden: Markt-Fundamentalismus ist in Deutschland wie auch in anderen europäischen Ländern sehr unpopulär geworden. Die deutschen Fundamentalisten begeben sich deshalb in einen verblüffenden Spagat zwischen Rhetorik und praktischer Politik. Das Vehikel dafür heißt „Staatsverschuldung".

Bevor ich mich diesem alles beherrschenden Thema widme, sollen kurz die offensichtlichsten Anzeichen einer Rückkehr zur wirtschaftlichen Grundkonstellation der Zeit vor der Krise benannt 
werden, die in hohem Maße von dem geprägt war, was Ökonomen als „Beggar thy Neighbour“-Politik bezeichnen: Eine Volkswirtschaft verschafft sich Vorteile zulasten ihrer Nachbarn. Gewöhnlich tun dies kleine Länder und richten damit keinen Schaden an. Doch in der Eurozone war es ausgerechnet die größte Volkswirtschaft, die vor der Krise diesen Weg beschritten hat. Nach dem Absturz tut sie dies erneut, wenn auch mit teilweise anderen Mitteln, und sie begleitet dies mit der drohenden (allerdings zum Scheitern verurteilten) Belehrung, dass alle anderen ihrem Vorbild zu folgen hätten.

\section{1 „BEGGAR AND LECTURE THY NEIGHBOUR" MIT GEGENWIND}

Verschiedene Indikatoren zeigen auf precrisis reloaded: An dem bemerkenswerten Wachstum seit Ende 2009, das jetzt allerdings in den „Abwärtssog“ der Krise im Euroraum gerät (IMK Arbeitskreis Konjunktur 2011), hatten die staatlichen und privaten Konsumausgaben erneut einen geringen Anteil. Der Anstieg der Lohnquote während des wirtschaftlichen Absturzes wird jetzt wieder "korrigiert" (vgl. Schäfer in diesem Heft). Der Anteil der Beschäftigten und der Haushalte mit niedrigen Löhnen ist leicht über das Vorkrisenniveau hinaus gewachsen (Bosch 2011). Die Zahl der Beschäftigten in Leiharbeit lag im Sommer 2011 um 100.000 über dem vor der Krise erreichten Höchststand (IW 2011). Und nicht zuletzt werden die Arbeitszeiten über den durch den Abbau der Kurzarbeit ausgelösten Effekt hinaus wieder länger (IAQ 2011). Bereits nach sehr kurzer Zeit bewahrheitet sich die Voraussage von Bosch (2011, S. 282), dass die „Dualisierung des Arbeitsmarkts vor der Krise nicht so offensichtlich war, aber im Aufschwung sichtbarer werden kann."

Doch die Hindernisse für die Fortsetzung dieses Kurses werden größer. Zum einen aus innenpolitischen und binnenwirtschaftlichen Gründen. In vielen Industrieunternehmen wachsen die Sorgen vor einem zukünftigen Fachkräftemangel. Zwar ist umstritten, wie begründet diese Sorgen sind. Doch ebenso wie die Probleme, die angesichts des demografischen Wandels mit älter werdenden Belegschaften verbunden sind, geben solche Befürchtungen denjenigen Rückenwind, die nachhaltigen Personalstrategien den
Vorzug geben vor dem kurzfristigen Ausnutzen von Lohndifferentialen in dualisierten Arbeitsmärkten. Auch politische Hindernisse werden größer. Der Druck der Gewerkschaften für unkonditioniertes „equal pay“ in der Leiharbeit und für einen gesetzlichen Mindestlohn erfreut sich wachsender Zustimmung in der Öffentlichkeit. Bis auf Weiteres ändert dies zwar nichts an den oben beschriebenen Faktoren, die zum Anwachsen des Niedriglohnsektors und dem Stagnieren oder gar Sinken der durchschnittlichen Arbeitseinkommen geführt haben. Dies umso weniger, als eine besonders große Hypothek für die zukünftige Entwicklung der Löhne, die sogenannten Hartz-Reformen, von keiner der gegenwärtigen oder potenziellen Regierungsparteien infrage gestellt wird. Dennoch ist es absehbar, dass die Lohndämpfungs-Strategie in Zukunft nicht ganz so reibungslos verfolgt werden kann wie vor der Krise.

Außenwirtschaftliche Gründe kommen hinzu. Die deutsche Regierung hat seit 2009 „Lohndämpfung“ mit großem Erfolg zu einem Exportschlager gemacht. Andere Länder werden gezwungen, sich am deutschen Beispiel zu orientieren, damit sie ihre Wettbewerbsfähigkeit erhöhen und ihre Staatsschulden reduzieren können. Das Problem dabei ist zumindest innerhalb der Eurozone, dass, wenn alle dem deutschen Beispiel folgten, die bisherige deutsche Strategie des Exportüberschusses nur um den Preis eines noch stärkeren Drucks auf die Löhne und einer noch stärkeren Ausweitung des Niedriglohnsektors in Deutschland weiterverfolgt werden könnte. Und ob genau dies innenpolitisch durchsetzbar wäre, ist fraglich. Paradoxerweise ist deshalb die deutsche Politik des beggar and lecture thy neighbour gegenwärtig dabei, die Voraussetzungen ihrer bisherigen Erfolge zu untergraben.

All dies vollzieht sich vor dem Hintergrund der ruckartig angestiegenen Staatsverschuldung.

\subsection{DER RETTUNGSANKER DES NEOLIBERALISMUS}

„Die Krise begann mit zu hohen und gehebelten privaten Schulden, die zu staatlichen Haushaltsdefiziten und Schulden wurden, weil die Rezession die Verschlechterung der Haushaltslage auslöste und ein großer Teil der privaten Schulden durch Rettungsaktionen für das Finanzsystem so- zialisiert wurden" (Roubini 2010). Obwohl dieser Zusammenhang und diese Abfolge der Ereignisse weithin unbestritten sind, wird das Opfer schuldig gesprochen. Die Reduzierung der staatlichen Defizite und Schulden (und zwar schwerpunktmäßig durch Senkung der Ausgaben! ) wird als Schlüssel für alles angesehen: Kurzfristig soll der Schuldenabbau der Abwehr spekulativer Attacken auf Staatsanleihen von "Schuldensündern“ dienen; mittelfristig schaffe er das „Vertrauen“, das für die wirtschaftliche Erholung nötig sei; langfristig sei er der einzige Weg, der „unsere Kinder und Enkel“ davor bewahre, die Kosten dafür zu tragen, dass „wir heute über unsere Verhältnisse leben“. Bei aller EuropaRhetorik wird dieses Argumentationsmuster flankiert von nationalem Egoismus. Wenn die Indignados in Madrid oder Athen ihren Banken und Regierungen zurufen, „Wir zahlen nicht für eure Krise!“, dann wird den Deutschen von ihrer Regierung und einigen Leitmedien - im besten Falle nur implizit - nahegelegt zurückzurufen: „Wir zahlen nicht für eure Schulden!“. Die Struktur dieses Gedankens ist im Kern eine ähnliche wie die des Rechtspopulismus in anderen europäischen Ländern. ${ }^{4}$ Sie bildet einen zusätzlichen ideologischen Kitt für eine Fokussierung jeglicher Wirtschaftsund Sozialpolitik auf die Reduzierung der Staatsausgaben durch „Einsparungen“.

Diese Fokussierung ist zum Rettungsanker für den Kern des Neoliberalismus geworden: Die neoliberale Politik einer Verstümmelung des Öffentlichen wird auf diese Weise mit neuer Begründung verschärft fortgesetzt. Dass alle wirtschaftlichen Ungleichgewichte, auch die in den Leistungsbilanzen, verschwinden würden, sobald die Staatshaushalte ausgeglichen seien, ist ein, wie es ein Kolumnist der Financial Times formuliert hat, „quasi-religiöser Glaube“ (Wolf 2011) der führenden Kräfte der Eurozone, der einzelnen Mitgliedsländern notfalls auch durch massives Hineinregieren aufgezwungen wird.

Als Beispiel mag folgende Erklärung der Vorsitzenden des französischen Front National vom Sommer 2011 dienen: „Wir haben bei uns genügend Armut, Elend und Arbeitslosigkeit, als dass wir -zig Milliarden französische Euro, die von Franzosen erarbeitet worden sind, anderen Ländern geben könnten. Wir haben selber genügend Schulden, um uns zu weigern, auch noch die der anderen zu bezahlen. Die Deutschen haben das gut verstanden und weigern sich jetzt, den anderen Ländern noch mehr unter die Arme zu greifen “ (Le Pen 2011) 
Letzteres ist genau das, was gegenwärtig in der Eurozone passiert. Auf Druck insbesondere der deutschen Bundesregierung wird ein Regime der Durchsetzung von Austeritätspolitik in den sogenannten Peripherie-Ländern der Eurozone geschaffen, das jeglicher demokratischer Legitimation, sei es auf EU-Ebene, sei es in den beteiligten Ländern, entbehrt (Leschke et al. 2012). Wenn dabei der Anschein erweckt wird, als könne Deutschland als Vorbild für alle dienen, dann lebt die Überzeugungskraft dieses Vorgehens teilweise erneut von der Krise der anderen. Zwar ist auch die deutsche Staatsschuld im Zusammenhang mit der Krise sprunghaft angestiegen (sie entspricht in Ausmaß und erreichtem Niveau ungefähr dem Durchschnitt aller Mitgliedsländer der Eurozone). Doch ist die Neuverschuldung bislang weniger rasch angestiegen als ursprünglich befürchtet, weil das Wachstum der Wirtschaft wie auch der Steuereinnahmen in den Jahren 2009 und 2010 überraschend kräftig war. Auf diese Weise wurde ein gewisser positiver Wachstumsbeitrag der Staatsausgaben im Jahre 2010 ermöglicht - ein Effekt, der anderen Ländern mit Hinweis auf die Notwendigkeit des sofortigen Abbaus der Neuverschuldung verwehrt wird. Hinzu kam eine kaum beachtete Nebenwirkung der fortgesetzten Krise in der Eurozone: Deutsche Bundesanleihen wurden ein so begehrtes Anlageobjekt, dass ihre Zinsen von knapp $5 \%$ vor der Krise mittlerweile auf Werte gesunken sind, die teilweise unter der Inflationsrate liegen. Deutschland verschuldet sich, wenn man so will, gegenwärtig real zum Nulltarif. Dieser „safe haven effect“ (Deutsche Bundesbank 2011b, S. 42) hat den Anstieg des Haushaltsdefizits der Bundesrepublik zusätzlich verringert und ist nichts anderes als eine Art Krisendividende (was in der deutschen Öffentlichkeit selbstverständlich nicht zur Kenntnis genommen wird).

Diese Faktoren erleichtern es der deutschen Politik, die Kürzungspolitik im eigenen Land zunächst noch etwas zu entdramatisieren. Doch das wird nicht so bleiben. Bereits jetzt betreffen die eingeleiteten Konsolidierungsmaßnahmen schwerpunktmäßig die Sozialausgaben (Heise/ Lierse 2011). Im weiteren Verlauf des jetzigen Jahrzehnts wird die in Deutschland mit Zustimmung aller aktuellen und potenziellen Regierungsparteien eingeführte Schuldenbremse, die nach dem Willen der deutschen Politik zum Allgemeingut in den Euroländern werden soll, als Fessel für zukünftiges Regierungshandeln immer schmerzhafter spürbar werden (Truger/ Teichmann 2010). Die öffentliche Finanznot, die heute vor allem auf der Ebene der Länder und der Kommunen zu spüren ist, wird die Möglichkeiten des Staates zunehmend beeinträchtigen, die für die Entwicklung der endogenen Potenziale dieses Landes erforderlichen Investitionen nicht allein in Beton, sondern vor allem in Menschen zu tätigen (vgl. dazu Vester in diesem Heft).

Doch diese Probleme wirken harmlos im Vergleich zu denen in den Ländern der Euro-Peripherie (vgl. die einschlägigen Länderanalysen in Lehndorff 2012). Dort bewirkt die Kürzungspolitik derzeit eine massive Vertiefung der Ungleichheit und stellt kurz- bis mittelfristig eine ernste Bedrohung für die wirtschaftliche Erholung und das Zurückdrängen der Arbeitslosigkeit dar. Mehr noch: Im Kern erschwert sie die Schaffung von Grundlagen für die längerfristige Stärkung der Wertschöpfungsbasis in diesen Ländern, für die ein leistungsfähiger Staat mit starker Steuerbasis benötigt wird.

\subsection{HAUSAUFGABEN - AUCH FÜR DEUTSCHLAND}

Die oktroyierte Austeritätspolitik ist eine Perversion des ursprünglichen Gedankens einer europäischen Wirtschaftsregierung, denn unter den Bedingungen der Währungsunion würde eine demokratisch legitimierte Wirtschaftsregierung heute notwendig auf einer Art europäischer „Ausgleichsunion“ (Troost/Paus 2011) beruhen: Überschüsse der einen würden strategisch eingesetzt, um andere leistungsfähiger zu machen (zur Diskussion über die dringendsten Reformen in der Eurozone vgl. Degryse/Pochet 2011). Diese strategische Herausforderung ist zweifellos ungeheuer groß, aber wenn der Euro tatsächlich eine Zukunft als europäische Binnenwährung haben soll, ist sie ist die wirtschaftspolitisch logische Konsequenz (Horn et al. 2011).

Selbstverständlich kann diese Rechnung nur aufgehen, wenn in den Ländern der Euro-Peripherie tatsächlich solche grundlegenden Reformen eingeleitet werden, mit denen die Voraussetzungen für nachhaltige Wachstumsmodelle (oder, um es weniger kontrovers zu formulieren, sozial-ökologische Entwicklungsmodelle) geschaffen werden (vgl. dazu Hübner in diesem Heft). Kritische Wirtschaftswissenschaftlerinnen und -wissenschaftler aus den betreffenden Ländern sehen dies ebenso. So fragt die italienische Ökonomin Annamaria Simonazzi (2012), ob es, erstens, „ein gemeinsames Interesse gibt, das Überschuss- und Defizitländer verbindet“, und zweitens, „welche Reformen die südlichen Länder in eigener Regie einleiten müssen, um der gemeinsamen Währung zu einer tragfähigen Grundlage zu verhelfen“. Ihre mutige Schlussfolgerung: „Erst wenn wir unsere Hausaufgaben gemacht haben, können wir Europas Hilfe verlangen.“

Dieses Argument hat jedoch eine weithin unbeachtete Pointe: Es gilt in anderem Sinne ebenso für Deutschland. Wenn es richtig ist, dass der neoliberale Umbruch im deutschen Modell des Kapitalismus zu den Wurzeln der wirtschaftlichen Krise in Europa gehört und auch gegenwärtig die Überwindung der Krise erheblich erschwert, dann sind Reformen in Deutschland für einen Ausweg aus der Krise ebenso wichtig wie Reformen in den Defizitländern. $\mathrm{Zu}$ den wichtigsten Elementen einer grundlegenden Reform des deutschen Beschäftigungsmodells - und zwar einer Reform, die diesem Begriff seine verloren gegangene Konnotation zurückgeben würde - gehört

- erstens, eine Stärkung der öffentlichen Investitionen und sozialen Dienstleistungen, die ohne deutliche Erhöhungen der Steuern auf Gewinne und höhere Einkommen nicht möglich sein wird;

- zweitens, eine geschlechterpolitische Modernisierung des Wohlfahrtsstaates;

- und drittens, eine Stützung des geschwächten Tarifvertragssystems durch gesetzlichen Mindestlohn, erweiterte Allgemeinverbindlicherklärungen von Tarifverträgen, unkonditioniertes equal pay bei Leiharbeit sowie die Bindung öffentlicher Aufträge an das Einhalten von (allgemeinverbindlichen) Tarifverträgen.

Derartige Reformen würden im Übrigen für den Staatshaushalt ein Positivsummenspiel von wachsenden Einnahmen, verbesserten Dienstleistungen und Abbau von Ungleichheit auslösen. Deshalb gilt: Das wirtschaftlich stärkste Land Europas wird erst zum Motor der Integration, wenn es seine eigenen Hausaufgaben macht. 


\section{Ausblick: Dünnes Eis}

Das neue deutsche Kapitalismusmodell, das de facto ein Hybrid ist aus einigen erhalten gebliebenen Bestandteilen des „Rheinischen Kapitalismus", neoliberal inspirierten Umbauten und Demontagen dieses klassischen Modells in den zurückliegenden 20 Jahren sowie einem hartnäckigen geschlechterpolitischen Konservatismus, wird gegenwärtig im In- und Ausland als Erfolgsmodell gefeiert. Doch es ist weitaus fragiler und störanfälliger, als es seine Befürworter glauben machen. Eine Währungsunion, die dieses Modell als Vorbild akzeptiert und die politische Dominanz seiner führenden Vertreter zulässt, bewegt sich auf sehr dünnem Eis.
Das Paradoxe daran ist: Deutschland, seine Wirtschaft und seine Gesellschaft, haben das Potenzial, eine wesentlich positivere Rolle in Europa zu spielen. Die deutsche Industrie könnte ihre Stärken auf dem Feld der ökologischen Erneuerung entwickeln, getragen von einer vergleichsweise breiten Zustimmung in der Gesellschaft zum nachhaltigeren Umgang mit natürlichen Ressourcen. Mehr sozialer Ausgleich innerhalb der größten europäischen Volkswirtschaft würde den permanenten Sozialabbau- und Lohnsenkungsdruck auf andere Länder der Region und insbesondere der Währungsunion verringern. Deutschland könnte Motor einer sozial und ökologisch nachhaltigeren Neuorientierung in der EU werden und anderen Ländern mehr Luft zum Atmen geben, ihre eigenen Wirtschafts- und Sozialmodelle zu erneuern.
Dies wäre nicht zuletzt für die Demokratie in der EU und in ihren Mitgliedsländern ein großer Gewinn.

Doch das ist Zukunftsmusik. Einstweilen triumphieren die gescheiterten Ideen ein weiteres Mal. Die seltsame Basis dieses Triumphs ist die wegen des Vertrauens in diese Ideen sprunghaft angestiegene Staatsverschuldung, und sein Vehikel ist das der Eurozone oktroyierte Austeritätsregime. Daran zeigt sich, dass die gescheiterten Ideen diesmal nicht als Befreiungs- und Heilsversprechen daherkommen, sondern ihr neuerlicher Triumph auf Einschüchterung und Angst beruht. Dies zeugt immerhin von Realismus, denn die Politik des NichtLernens kann nur weitere Krisen hervorbringen. Bleibt zu hoffen, dass nicht auch das europäische Projekt zu ihren nächsten Opfern gehört.

\section{LITERATUR}

Bispinck, R. (2011): Pay in the 2000s: development and outcomes, WSI Report (6), Düsseldorf

Bispinck, R./Schulten, T. (2009): Re-Stabilisierung des deutschen Flächentarifvertragssystems, in: WSI-Mitteilungen 62 (4), S. 201-209 Bofinger, P. (2008): Das Jahrzehnt der Entstaatlichung, in: WSI-Mitteilungen 61 (7), S. $351-357$

Bofinger, P. (2010) Eine andere Meinung zur Rolle Deutschlands in der Europäischen Währungsunion, in: Sachverständigenrat zur Begutachtung der gesamtwirtschaftlichen Entwicklung, Jahresgutachten 2010/11, S. 123-133

Bosch, G. (2011): The German labour market after the financial crisis: Miracle or just a good policy mix?, in: Vaughan-Whitehead, D. (Hrsg.): Inequalities in the world of work: The effects of the crisis, International Labour Office, Genf, S. 255-286

Bosch, G./Kalina, T. (2008): Low-wage work in Germany: an overview, in: Bosch, G./Weinkopf, C. (Hrsg.): Low-wage work in Germany, Russell Sage Foundation, New York, S. 19-112

Brenke, K. (2011): Einkommensumverteilung schwächt privaten Verbrauch, DIW-Wochenbericht (8), S. 2-12

Bundesagentur für Arbeit (BA) (2011): Zeitarbeit in Deutschland - Aktuelle Entwicklungen, Bundesagentur für Arbeit, Nürnberg

Chakrabortty, A. (2011): Which is the No 1 problem economy in Europe?, Guardian, 8. August, Online: http://www.guardian.co.uk/ commentisfree/2011/aug/08/number-one-problem-economy-europe (letzter Zugriff: 25. August 2011)

Degryse, C./Pochet, P. (2011): Monetary Union, economic coordination and democratic legitimacy, European Trade Union Institute, ETUI Policy Brief (5), Brüssel, http://www.etui.org/Publications2/PolicyBriefs/European-Social-Policy/Monetary-Union-economic-coordination-and-democratic-legitimacy (letzter Zugriff: 26. Oktober 2011) Destatis (2009): Niedrigeinkommen und Erwerbstätigkeit, Statistisches Bundesamt, Wiesbaden, http://www.destatis.de/jetspeed/portal/cms/ Sites/destatis/Internet/DE/Presse/pk/2009/Erwerbstaetigkeit/
begleitheft__Erwerbstaetigkeit,property=file.pdf (letzter Zugriff: 25.

August 2011)

Destatis (2011): Jugenderwerbslosigkeit in Deutschland ist eine der niedrigsten in der EU, Statistisches Bundesamt, Pressemitteilung Nr. 293 vom 11.08.2011, http://www.destatis.de/jetspeed/portal/cms/ Sites/destatis/Internet/DE/Presse/pm/2011/08/PD11__293__132.psml (letzter Zugriff: 25. August 2011)

Deutsche Bundesbank (2011a): Zur Entwicklung der Ausfuhr in den vier großen EWU-Mitgliedstaaten seit Beginn der Währungsunion, Monatsbericht Juli, S. 17-38

Deutsche Bundesbank (2011b): Renditedifferenzen von Staatsanleihen im Euro-Raum, Monatsbericht Juni, S. 29-47

Deutsche Bundesbank (o.J.): Statistik Leistungsbilanz, http://www. bundesbank.de/statistik/statistik_zeitreihen.php?lang=de\&open= wirtschaftsdaten\%20\&func $=$ list\&tr=www_s311_b40301 (letzter Zugriff: 31. Oktober 2011)

Dullien, S./Schieritz, M. (2011): Die deutsche Investitionsschwäche: Die Mär von den Standortproblemen, in: Wirtschaftsdienst 91 (7), S. $458-468$

Ellguth, P./Kohaut, S. (2010): Auf der Flucht? Tarifaustritte und die Rolle von Öffnungsklauseln, in: Industrielle Beziehungen 17 (4), S. 345-371 Erlinghagen, M. (2010): Mehr Angst vor Arbeitsplatzverlust seit Hartz? Langfristige Entwicklung der Beschäftigungsunsicherheit in Deutschland, Institut Arbeit und Qualifikation, IAQ-Report (2), Duisburg Europäische Zentralbank (ECB) (2011): Harmonised Competitiveness Indicators, http://www.ecb.int/stats/exchange/hci/html/index.en.html (letzter Zugriff: 25. August 2011)

European Commission (2010): The impact of the global crisis on competitiveness and current account divergences in the euro area, European Commission, Directorate-General for Economic and Financial Affairs, Quarterly report on the Euro area 1 (9), Brüssel, http://ec.europa.eu/ economy_finance/publications/qr_euro_area/2010/pdf/qrea201001en. pdf (30.10.2011) 
Fricke, T. (2010): Viel Wind um wenig Wunder, Financial Times Deutschland vom 12. März

Fuchs, J./Hummel, M./Klinger, S./Spitznagel, E./Wanger, S./Zika, G. (2010): Der Arbeitsmarkt schließt an den vorherigen Aufschwung an, IAB-Kurzbericht (18), Nürnberg

Haipeter, T. (2009): Tarifabweichungen und Flächentarifverträge: Eine Analyse der Regulierungspraxis in der Metall- und Elektroindustrie, Wiesbaden

Heise, A./Lierse, H. (2011): Haushaltskonsolidierung und das Europäische Sozialmodell, Friedrich-Ebert-Stiftung, Berlin

Herzog-Stein, A./Lindner, F./Sturn, S./van Treeck, T. (2011): Vom Krisenherd zum Wunderwerk? Der deutsche Arbeitsmarkt im Wandel, IMK Report (56), Düsseldorf

Horn G./Dröge, K./Sturn, S./van Treeck, T./Zwiener, R. (2009): Von der Finanzkrise zur Weltwirtschaftskrise (III) - Die Rolle der Ungleichheit, IMK Report (41), Düsseldorf

Horn, G./Lindner, F./Niechoj, T. (2011): Schuldenschnitt für Griechenland - ein gefährlicher Irrweg für den Euroraum, IMK-Report (63), Düsseldorf

Horn, G./Logeay, C./Stephan, S./Zwiener, R. (2007): Preiswerte Arbeit in Deutschland, Auswertung der aktuellen Eurostat Arbeitskostenstatistik, IMK Report (22), Düsseldorf

Huffschmid, J. (2010): Fehlverhalten, Regulierungsmängel oder Systemdynamik? Zu den Hintergründen und Ursachen der Finanzkrise, in: Hickel, R./Troost, A. (Hrsg.): Kapitalismuskritik heute: Vom staatsmonopolistischen zum finanzmarktgetriebenen Kapitalismus, Hamburg, S. 21-34 ICTWSS Database (2011): Database on Institutional Characteristics of Trade Unions, Wage Setting, State Intervention and Social Pacts in 34 countries between 1960 and 2007, Amsterdam Institute for Advanced Labour Studies (AIAS), University of Amsterdam, http://www.uva-aias. net/208 (letzter Zugriff: 2. August 2011)

Institut der Deutschen Wirtschaft (IW) (2011): Wachstum in der Zeitarbeit setzt sich fort. IW-Zeitarbeitsindex, BAP-Umfrage September 2011, http://www.personaldienstleister.de/pdf/BAP-Bericht_Welle_ 46_20111011.pdf (letzter Zugriff: 30.10.2011)

Institut für Arbeit und Qualifikation (IAQ) (2011): Arbeitszeiten so lang wie vor der Krise, Pressemitteilung, Institut Arbeit und Qualifikation der Universität Duisburg-Essen, http://www.iaq.uni-due.de/archiv/ presse/2011/110902.php (letzter Zugriff: 25. Oktober 2011) Institut für Makroökonomie und Konjunkturforschung (IMK), Arbeitskreis Konjunktur (2011): Deutsche Konjunktur im Abwärtssog, IMK Report (65), Düsseldorf

International Labour Oganisation (ILO) (2010): Global Wage Report 2010/11: Wage policies in times of crisis, International Labour Office,

Genf

Joebges, H./Logeay, C./Stephan, S./Zwiener, R. (2010): Deutschlands Exportüberschüsse gehen zu Lasten der Beschäftigten, Friedrich-EbertStiftung, Bonn

Knuth, M. (2010): Fünf Jahre Hartz IV: Zwischenbilanz und Reformbedarf, in: Orientierungen zur Wirtschafts- und Gesellschaftspolitik (123), S. $14-23$

Krugman, P. (2010): When Zombies Win, New York Times vom 19. Dezember, http://www.nytimes.com/2010/12/20/opinion/20krugman. html?_r=1\&ref=paulkrugman\&pagewanted=print (letzter Zugriff: 25 . August 2011)

Le Pen, M. (2011): Conférence de presse exceptionnelle de Marine Le Pen sur la crise, 11. August, http://www.frontnational.com/?p=7240 (letzter Zugriff: 25. August 2011)
Lehndorff, S. (Hrsg.) (2009): Abriss, Umbau, Renovierung? Studien zum Wandel des deutschen Kapitalismusmodells, Hamburg Lehndorff, S. (2011): Before the crisis, in the crisis, and beyond: the upheaval of collective bargaining in Germany, in: Transfer 17 (3), S. 341-354

Lehndorff, S. (Hrsg.) (2012): A triumph of failed ideas. European models of capitalism in the crisis, European Trade Union Institute, Brüssel, http://www.etui.org/ (im Erscheinen)

Lehndorff, S./Bosch, G./Haipeter, T./Latniak, E. (2009): Vor der Krise und in der Krise: Das deutsche Beschäftigungsmodell - ein Modell für die ganze Welt?, in: Lehndorff, S. (Hrsg.): Abriss, Umbau, Renovierung? Studien zum Wandel des deutschen Kapitalismusmodells, Hamburg, S. $20-46$

Leschke, J./Theodoropoulou, S./Watt, A. (2012): How do economic governance reforms and austerity measures affect inclusive growth as formulated in the Europe 2020 strategy?, in: Lehndorff, S. (Hrsg.): A triumph of failed ideas (im Erscheinen)

Münchau, W. (2010): Hartz IV vergiftet Europa, Financial Times Deutschland vom 30. September

Niechoj, T./Stein, U./Stephan, S./Zwiener, R. (2011): Deutsche Arbeitskosten und Lohnstückkosten im europäischen Vergleich - Auswirkungen der Krise, IMK-Report (60), Düsseldorf

Organisation for Economic Co-operation and Development (OECD) (2010): Employment Outlook, Paris

Priewe, J./Rietzler, K. (2010): Deutschlands nachlassende Investitionsdynamik 1991-2010. Ansatzpunkte für ein neues Wachstumsmodell, Friedrich-Ebert-Stiftung, Bonn

Roubini, N. (2010): A Survival Strategy for the Eurozone, http://www. project-syndicate.org (letzter Zugriff: 25. August 2011)

Sachverständigenkommission (2011): Neue Wege - gleiche Chancen. Gleichstellung von Frauen und Männern im Lebensverlauf, Gutachten der Sachverständigenkommission an das Bundesministerium für Familie, Senioren, Frauen und Jugend für den ersten Gleichstellungsbericht der Bundesregierung, Berlin

Simonazzi, A. (2012): Italy - Still life, in: Lehndorff, S. (Hrsg.): A triumph of failed ideas (im Erscheinen)

Troost, A./Paust, L. (2011): Die Europäische Ausgleichsunion - Die Währungsunion 2.0, Institut Solidarische Moderne, Frankfurt a. M., http://www.solidarische-moderne.de/de/article/154.die-europaeischeausgleichsunion-die-waehrungsunion-2-0.html (letzter Zugriff: 30. Oktober 2011)

Truger, A./Teichmann, D. (2010): IMK-Steuerschätzung 2010-2014: Kein Spielraum für Steuersenkungen, IMK-Report (49), Düsseldorf Urban, H.-J. (2012): Crisis corporatism and trade union revitalisation in Europe, in: Lehndorff, S. (Hrsg.): A triumph of failed ideas (im Erscheinen)

Voss-Dahm, D. (2011): Die Bedeutung beruflicher Bildungsabschlüsse für die Karriere im Betrieb, in: Voss-Dahm, D./Mühge, G./Schmierl, K./Struck, O. (Hrsg.): Qualifizierte Facharbeit im Spannungsfeld von Flexibilität und Stabilität, Wiesbaden, S. 163-185

Weinkopf, C. (2009): Germany: precarious employment and the rise of mini-jobs, in: Vosko, L./MacDonald, M./Campbell, I. (Hrsg.): Gender and the Contours of Precarious Employment, London, S. 177-193 Weinkopf, C./Vanselow, A. (2008): (Fehl-)Entwicklungen in der Zeitarbeit?, Friedrich-Ebert-Stiftung, Bonn

Wolf, M. (2011): There is no sunlit future for the euro, Financial Times vom 19. Oktober 